\title{
On how non-magnetic particles intensify rotational diffusion in magnetorheological fluids
}

\author{
L. Rodríguez-Arco, ${ }^{*}$ M.T. López-López, ${ }^{*}$ P. Kuzhir, ${ }^{\ddagger}$ F. González-Caballero ${ }^{*}$
}

Receipt date: 29 March 2014

\begin{abstract}
In this work we propose a mechanism to explain the enhancement of the magnetic field-induced yield stress when non-magnetic particles are added to magnetic particulate suspensions -i.e., bi-component suspensions. Our main hypothesis is that the non-magnetic particles collide with the field-induced magnetic aggregates under shear flow. Consequently, supplementary fluctuations of the orientations of the magnetic aggregates occur, resulting in an effective rotary diffusion process, which increases the dynamic yield stress of the suspension. Furthermore, the collision rate and the rotary diffusivity of the aggregates should increase with the concentration of non-magnetic particles. Rheological measurements in plate-plate and cylindrical Couette geometries confirm the increase of the yield stress with the volume fraction of non-magnetic particles. In addition, such an effect appears to be more important in Couette geometry, for which orientation fluctuations of the magnetic aggregates play a more significant role. Finally, a theoretical model based on this rotary diffusion mechanism is developed, providing with a quantitative explanation to the experimentally-observed trends.
\end{abstract}

PACS: 66.10.C-; 47.11.-j; 83.80.Hj; 83.80.Gv.

\footnotetext{
* Departmet of Applied Physics, University of Granada, Faculty of Science, Campus de Fuentenueva, 18071 Granada, Spain.

† Corresponding author. Email address: 1_rodriguezarco@ugr.es

¥ University of Nice-Sophia Antipolis, CNRS, U.M.R 7336, Laboratory of Condensed Matter Physics, 28 avenue Joseph Vallot, 06100 Nice, France
} 


\section{INTRODUCTION}

The fluid dynamics of particles suspended in a liquid -i.e., particulate suspensions-have been extensively studied in recent decades due to their multiple applications. Special attention has been paid to diffusion, the process which governs the motion of the suspended particles, either by controlling the particles' position (translational diffusion) or their orientation (rotational diffusion) [1]. Typical fields where diffusion plays a crucial role are self-assembly and rheology/micro-rheology of complex fluids (polymer solutions, suspensions of rod-like particles or granular gases) [2-6]. A special kind of particulate suspensions for which diffusion has also been studied are field-driven colloids, suspensions of polarizable particles dispersed in a liquid carrier which undergo changes of their mechanical properties in the presence of external fields [7-12]. Examples of these smart materials are ferrofluids or electrorheological and magnetorheological (MR) fluids. In the case of MR fluids, the suspended magnetizable particles build columnar-like aggregates in the direction of the applied magnetic field. Such a jamming process induces a several orders of magnitude increase of the MR fluid viscosity in the presence of the field, a phenomenon commonly known as the MR effect [13-14].

The importance of rotational diffusion on the rheological (flow) properties of MR fluids is clearly evidenced when comparing such properties in the available rheometry configurations. In the particular case of a magnetic field applied in the direction perpendicular to the walls which confine the sample -for example, in plate-plate or cone-plate geometries- the magnetic aggregates span the gap between the geometry walls, hindering the rotation of the upper plate/cone upon the application of a given stress. Nevertheless, there is a threshold value of the stress, also known as the yield stress, for which these structures are broken, losing contact with the walls, so that a practical onset of the flow takes place. Such behavior is reminiscent of the Bingham plastic behavior, the yield stress being an increasing function of the magnetic field [13]. On the other hand, when the magnetic field is oriented parallel to the geometry walls e.g., cylindrical Couette geometry or pressure-driven flows using coaxial coils-, the aggregates are theoretically oriented along the stream-lines and have in theory an infinite length due to the absence of tensile hydrodynamic forces. Consequently, the suspension should not develop any yield stress and its rheological behavior should follow Newton's law of viscosity. However, experimental results show exactly the opposite effect: the suspension develops a strong Bingham behavior [15-18]. In a previous work [19] we have shown that the main contribution to the appearance of such a yield stress is precisely the rotational diffusion of the field-induced aggregates. More specifically, stochastic rotary oscillations of such aggregates increase the stress level of the suspension. These oscillations are caused by many-body magnetic interactions with neighboring aggregates [19].

However, and to the best of our knowledge, the effect of rotational diffusion on the yield stress has only been studied in the case of conventional MR fluids, that is, suspensions consisting solely of micron-sized ferromagnetic particles. However, in the last decades a number of methods to enhance the applicability of MR fluids -i.e., increasing the suspension stability and the field-induced yield stress- have been described. One effective way to improve stability is the use of non-magnetic -i.e., diamagnetic- particles in the formulation, such as clay, polymeric or silica particles [20]. Due to their lower density they contribute to a reduction of particle settling without increasing the final weight of the fluid. In addition to a better stability, its use results in an enhanced MR effect [21-25]. However, the physics behind such an increase still remains unclear. For example, López-López et al. [21] attributed the increase of the MR effect when dispersing iron and clay particles together to the combination of the 
magnetically-induced iron chains and a clay gel. Particle-level simulations in three dimensions supported the experimental enhancement of the yield stress when mixing iron particles and hollow glass beads [22]. However simulations in a monolayer of particles did not confirm the experimental trends. The authors themselves stated that a mechanistic explanation was still lacking [22]. In a second work, new simulations suggested that the non-magnetic particles increased the size of the field-induced clusters [23].

In a previous work we showed that the enhancement of the MR effect in bi-component suspensions could be attributed to a change in the magnetic properties of the suspension when iron particles -approx. $1 \mu \mathrm{m}$ in size-adsorbed onto a layer around poly(methylmethacrylate), PMMA, spheres - of approx. $10 \mu \mathrm{m}$ of diameter. Indeed, simulations showed that suspensions of such non-magnetic-core-magnetic-shell composites would develop higher magnetic permeability than those of solid magnetic particles, with the same concentration of magnetic material [25]. We have given experimental evidence of such an increase in a recent work too, in which the magnetic properties and the MR effect of nickel-coated ceramic particles and solidnickel particles were compared [26].

In this work we deal with bi-component suspensions in which adhesion between PMMA and iron particles is avoided by the use of a surfactant, but still, a strong enhancement of the MR effect appears. Therefore, in the present case, the explanation to the improvement of the MR effect could not come from a change of the magnetic properties as in refs. [25, 26], and finding an alternative explanation is the main aim of this work. Our main hypothesis is based on collisions between the non-magnetic particles and the field-induced aggregates of magnetic particles under shear flow. Such collisions may impart supplementary fluctuations of the positions and orientations of the magnetic aggregates. In order to prove our hypothesis, we perform rheological cylindrical Couette measurements, because the effects of orientation fluctuations are more clearly evidenced in this geometry. For comparison we also show the results of plate-plate rheological measurements. Finally we develop a theoretical explanation for the experimentally observed trends.

\section{MATERIALS AND METHODS}

We used spherical carbonyl iron particles (BASF, HS quality) and PMMA spheres (Microbeads, Spheromers10) as magnetic and non-magnetic particles respectively. Particle diameters were $1.0 \pm 0.7 \mu \mathrm{m}$ and $9.9 \pm 0.4 \mu \mathrm{m}$ respectively. In order to hinder adsorption of iron particles around PMMA spheres we first dispersed appropriate amounts of iron powder in silicone oil (VWR International, Rhodorsil 47V500, dynamic viscosity at $25^{\circ} \mathrm{C}$ is $480 \mathrm{mPa} \cdot \mathrm{s}$ ) followed by the addition of aluminum stearate (Sigma Aldrich, technical grade), under vigorous mechanical stirring. We continued stirring for several hours to promote stearate adsorption onto iron, and finally added PMMA powder in appropriate amounts. The volume fraction of iron, $\Phi_{m}$, was $10 \mathrm{vol} \%$ for the four prepared samples. The volume fraction of PMMA, $\Phi_{n}$, ranged from 0 to $30 \mathrm{vol} \%$. All the samples were degasified under vacuum for 15 minutes prior to rheological measurements.

Microscopic observations upon magnetic field application of diluted samples, prepared as described above, were conducted by placing an optical microscope between two Helmholtz coils that applied a homogeneous magnetic field parallel to the surfaces that confined the sample. Magnetization curves of the suspensions were obtained at $20^{\circ} \mathrm{C}$ by means of a vibrating sample magnetometer VSM 4500 (EG\&G Princeton Applied Research, USA). 
The rheological measurements were conducted by using a controlled-stress rotational rheometer, Haake RheoStress RS 150 (Thermo Fisher Scientific, USA). We performed measurements using cylindrical Couette and plate-plate geometries. Couette cell consisted of an inner cylinder of diameter of $20 \mathrm{~mm}$ and height of $30 \mathrm{~mm}$. The radial distance between the surfaces of the outer and inner cylinders was $0.75 \mathrm{~mm}$. We applied a uniform magnetic field with the help of a coil placed coaxially with the rheometer axis and thus, in the direction parallel to the suspension vorticity. The measuring protocol for Couette measurements was as follows: (i) Pre-shear stage at a shear rate of $150 \mathrm{~s}^{-1}$ for $60 \mathrm{~s}$ in the absence of field. (ii) Application of the same shear rate for $60 \mathrm{~s}$ upon a magnetic field of approx. $6 \mathrm{kA} / \mathrm{m}$. (iii) Sample at rest for 3 min and magnetic field application of a desired intensity (from 6 to $30.6 \mathrm{kA} / \mathrm{m}$ ). (iv) Shear rate ramp (equivalent rheological results were obtained by ramping the shear stress) from 1 to 500 $\mathrm{s}^{-1}$ upon the same field as in (iii) with duration of each step of $30 \mathrm{~s}$. At the end of stage (iv), the magnetic field was again readjusted to $6 \mathrm{kA} / \mathrm{m}$, and stage (ii) was repeated before a new shear rate ramp at a different -increasing- magnetic field was started. Note that a magnetic field of, at least, $6 \mathrm{kA} / \mathrm{m}$ was maintained during the whole process to reduce particle settling.

Plate-plate measurements were performed with a set of parallel plates (diameter of 35 $\mathrm{mm})$. The gap between the two plates was $350 \mu \mathrm{m}$. All the quantities reported hereinafter correspond to the outer radial edge of the plate. In this second case, the magnetic field was applied with the same coil as for cylindrical Couette geometry. As a result, in this geometry the magnetic field was aligned along the velocity gradient and perpendicular to the rheometer walls. The measuring protocol consisted of three stages: (i) Pre-shear at a shear rate of $150 \mathrm{~s}^{-1}$ for 60 s. (ii) Sample at rest for $120 \mathrm{~s}$. (iii) Shear rate ramp from 20 to $300 \mathrm{~s}^{-1}$. Each step lasted $30 \mathrm{~s}$. The magnetic field was activated at the beginning of (ii) and was kept switched on until the end of (iii).

\section{EXPERIMENTAL RESULTS}

First of all, and to discard the formation of non-magnetic-core-magnetic-shell composites of enhanced magnetic permeability -i.e., like those of ref. [25] - we performed microscopic observations and magnetization measurements. The microscopy results obtained for diluted suspensions showed that there was not adsorption of iron particles onto PMMA ones. As a matter of fact, PMMA particles appeared uncovered and most of them were separated from the field-induced iron aggregates upon the application of an external magnetic field -Fig. 1 . The thickness of these chain-like aggregates was of the same order of magnitude as the diameter of PMMA particles. In addition, non-magnetic particles were generally not trapped into the aggregates of magnetic particles in contrast to the results shown in the inset of Fig. 1 for which aluminum stearate was not used and consequently, a strong cohesion between iron and PMMA particles existed, resulting in an increase of the MR effect with a growing content of PMMA [25]. Regarding the magnetization curves, we observed that the hysteresis loops for the different samples were practically superimposed, as seen in Fig. 2, and therefore, the addition of PMMA particles did not affect the suspension magnetic permeability. Actually, calculations of such magnetic permeability by using the ascent branch of the hysteresis loops (inset of Fig. 2) revealed differences in the magnetic permeability no bigger than approx. 5\% for the different samples. Therefore, changes of the MR effect for these bi-component suspensions could not be attributed to an enhancement of the magnetic permeability because of the formation of a magnetic coating around PMMA particles. 


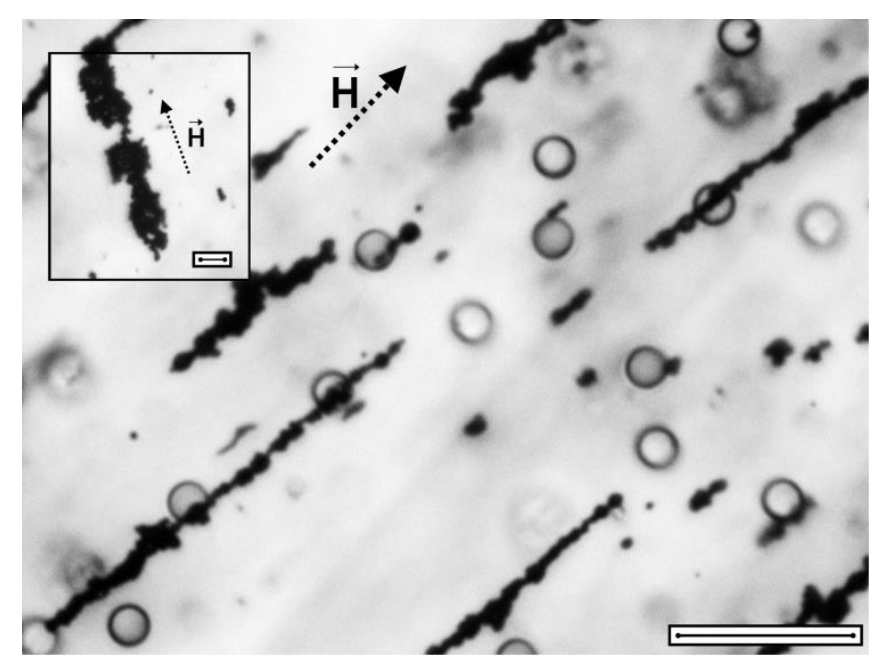

FIG. 1. Microscopic picture of a suspension of 1 vol \% iron and 3 vol \% PMMA dispersed in silicone oil and stabilized by the addition of aluminum stearate. A magnetic field of approx. $10 \mathrm{kA} / \mathrm{m}$ was applied in the direction indicated by the arrow; the bar length corresponds to $50 \mu \mathrm{m}$. PMMA particles (white spheres of 10 $\mu \mathrm{m})$ appeared uncoated and separated from the iron chains. This situation was different to that observed in the inset for a suspension in which aluminum stearate had not been added to the suspension and in which adsorption of iron particles around PMMA particles took place with the formation of non-magnetic-core-magnetic-shell composites. The picture from the inset is taken from ref. [25] and the bar length is $10 \mu \mathrm{m}$.

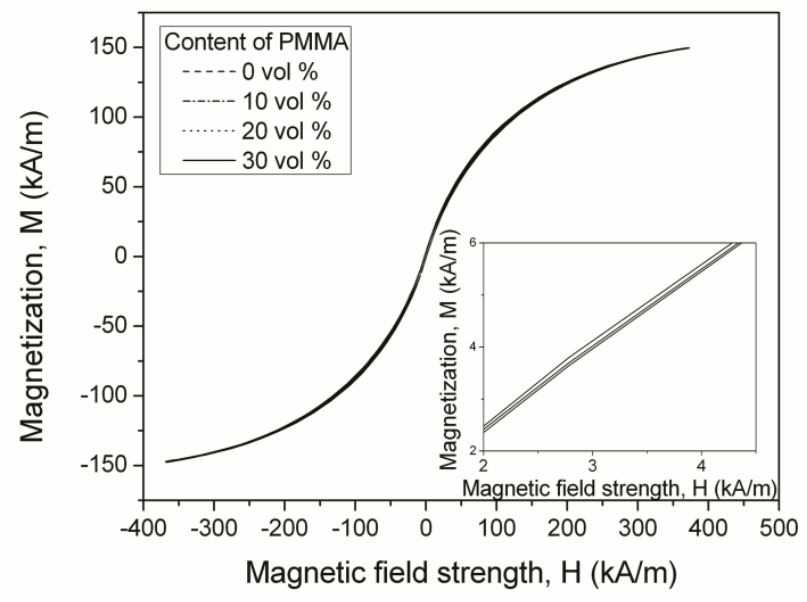

FIG. 2. Hysteresis loops for all the suspensions. The concentration of iron particles was 10 vol $\%$ while the volume fraction of PMMA particles ranged from 0 to $30 \mathrm{vol} \%$. All the curves were superimposed and therefore, there were no remarkable differences in the suspension magnetic permeability of the samples, calculated from the ascent branch of the inset.

However, and despite having removed the influence of an enhanced suspension permeability, we observed a strong enhancement of the MR effect, which seemed to be especially important in cylindrical Couette geometry. More specifically, the shear stress in the flow curves - shear stress $\sigma$ vs. shear rate $\dot{\gamma}$ - at given values of the external magnetic field and $\dot{\gamma}$, was higher when non-magnetic particles were included in the formulation for both cylindrical Couette and plate-plate geometries. In addition, $\sigma$ increased with the volume fraction of PMMA particles $\Phi_{n}$ for a certain value of $\dot{\gamma}$-Fig. 3. Note that the values of the shear stress for a particular suspension were higher when using cylindrical Couette geometry in comparison with the plate-plate geometry-Fig. 3. 
a)

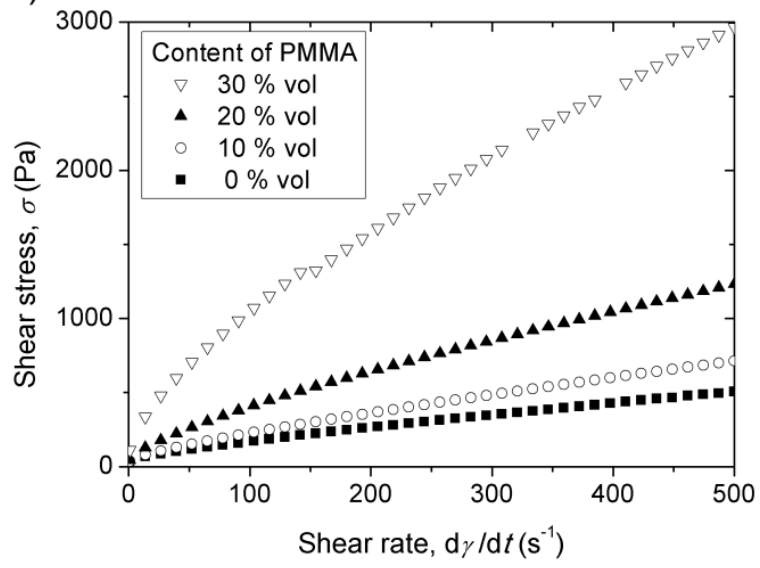

b)

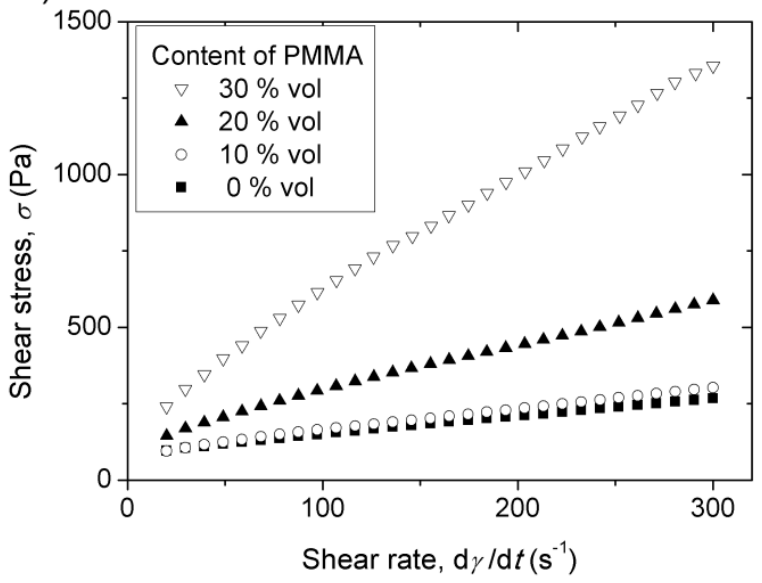

FIG. 3. Flow curves of the bi-component suspensions in cylindrical Couette (a) and plate-plate (b) geometries at the same concentration of iron particles, $\Phi_{m}=0.1$ and different concentrations of PMMA particles $\Phi_{n}$. The intensity of the external magnetic field was $H_{0}=12.2 \mathrm{kA} / \mathrm{m}$ for both geometries. As observed, the shear stress increased when $\Phi_{n}$ was increased. Note that the shear stress was generally higher for cylindrical Couette geometry.

In order to better compare both geometries and analyze the influence of the addition of PMMA on the MR effect, we estimated the yield stress. Recall that the MR effect is defined as the change of the rheological behavior from an almost Newtonian behavior in the absence of field -characterized by the equation $\sigma=\eta \dot{\gamma}$ where $\eta$ is the viscosity- to a plastic behavior when the magnetic field is activated. A plastic fluid usually follows Bingham's equation $\sigma=\sigma_{Y}+\eta \dot{\gamma}$ where $\sigma_{Y}$ is the dynamic yield stress [27]. We estimated the yield stress by performing a linear fit of the high-shear part of the rheograms $\left(\dot{\gamma}>100 \mathrm{~s}^{-1}\right)$, the yield stress being the intercept of the fit with the Y-axis, i.e., zero shear rate. The fits (not shown here for brevity) were reasonably good in all cases $\left(\mathrm{R}^{2} \approx 0.99\right)$. With the aim of just comparing the influence of the magnetic field between both geometries, we calculated the increment of the yield stress by subtracting the yield stress at zero field to the yield stress at a given applied field, and plotted it against the external magnetic field strength, $H_{0}$-Fig. 4. The so-estimated increment of the yield stress increased both with $H_{0}$ and $\Phi_{n}$ for plate-plate and cylindrical Couette geometries. In cylindrical Couette geometry, however, both effects appeared more intensified. Actually, the yield stress increment exhibited a stronger increase with the PMMA concentration in cylindrical Couette geometry -3.7 times in the range $0<\Phi_{n}<0.3$ - as compared to the plate-plate geometry -1.9 times in the same range. 

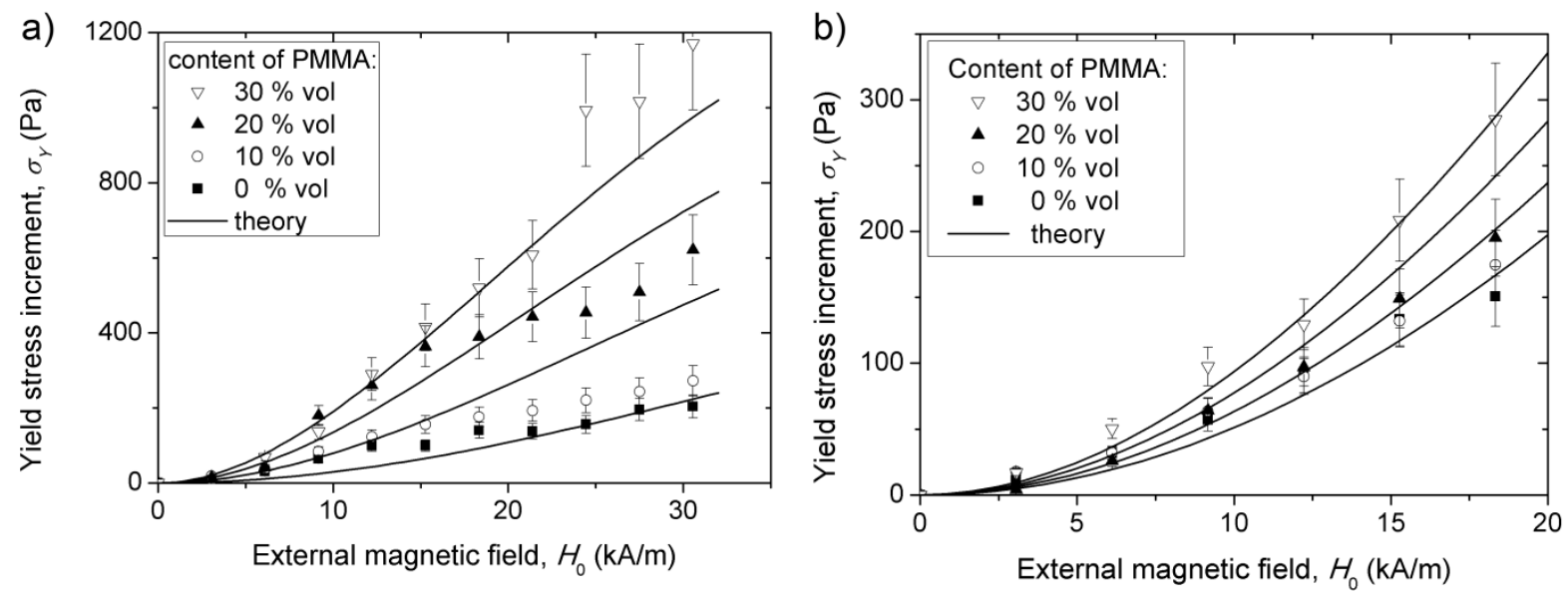

FIG.4. Experimental (symbols) and theoretical (lines) dependencies of the yield stress increment on the intensity of the external magnetic field for the cylindrical Couette (a) and the plate-plate (b) geometries at the same concentration of iron particles, $\Phi_{m}=0.1$ and different concentrations of PMMA particles, $\Phi_{n}$. The yield stress increased with both the magnetic field strength and the volume fraction of PMMA. Both effects appeared to be more pronounced in Couette rheometry. The theoretical predictions (see section IV) are obtained for values of the free parameters of $\alpha_{1}=1.5 ; \alpha_{2}=0.2$ for the plate-plate geometry, and $\alpha_{1}=0.2 ; \alpha_{2}=0.4$ for the cylindrical Couette geometry. The collision contribution to the rotary diffusivity $\alpha_{2}$ is thus more important in the second case.

To be precise, the differences between the increments of the yield stress for both geometries became more accentuated when the PMMA volume fraction increased. Indeed, the Couette yield stress increment of the sample without PMMA was almost superimposed to the equivalent plate-plate one, whereas the yield stress increment of the sample with $30 \mathrm{vol} \%$ PMMA was much higher for cylindrical Couette geometry -Fig. 5. However, we must keep in mind that because of the differences in geometry, the demagnetizing field opposed by the sample was different for the cylindrical Couette and the plate-plate systems. In the Couette geometry the height-to-gap ratio was very large and, as a result, the demagnetizing field along the rheometer axis was negligible. Consequently the field inside the sample, the so-called internal field, $H$, was almost equal to the external one $H \approx H_{0}$. On the other hand, in plate-plate rheometry, the ratio of the gap height to the plate diameter was small and the resulting demagnetizing field was higher. Estimations of the internal magnetic field using Eq. (A1) show that for the sample without PMMA, the MR effect in plate-plate geometry was considerably higher than in the case of cylindrical Couette. However, the differences between both geometries diminished as the PMMA concentration increases because of the further enhancement of the MR effect in bi-component suspensions for cylindrical Couette geometry (See Supplemental Material at [URL will be inserted by publisher] for the comparison between both geometries as a function of $H$ ). 
a)

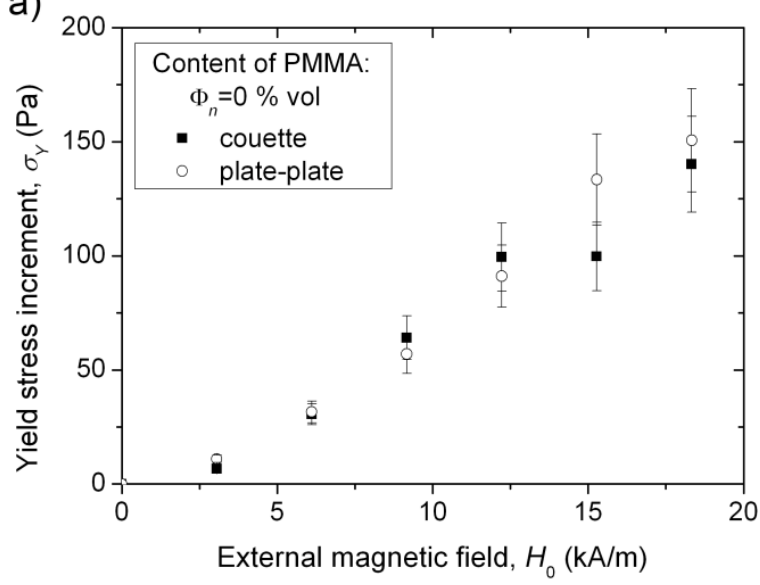

c)

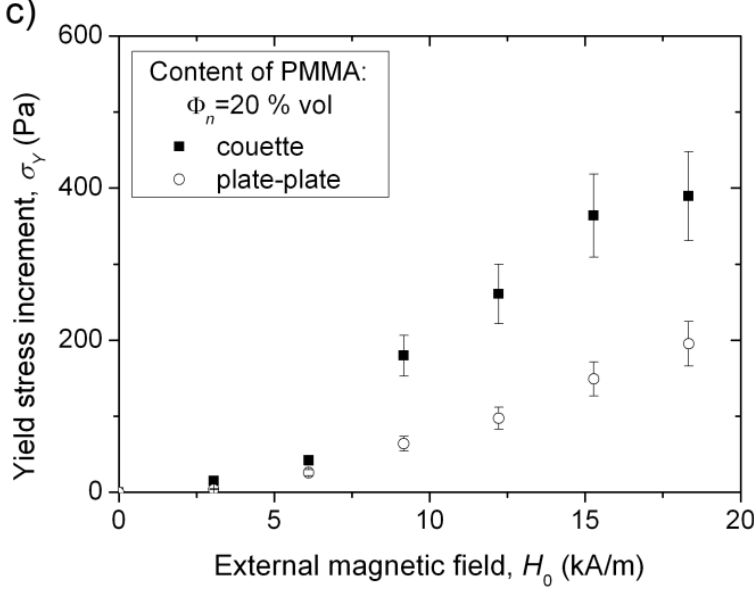

b)

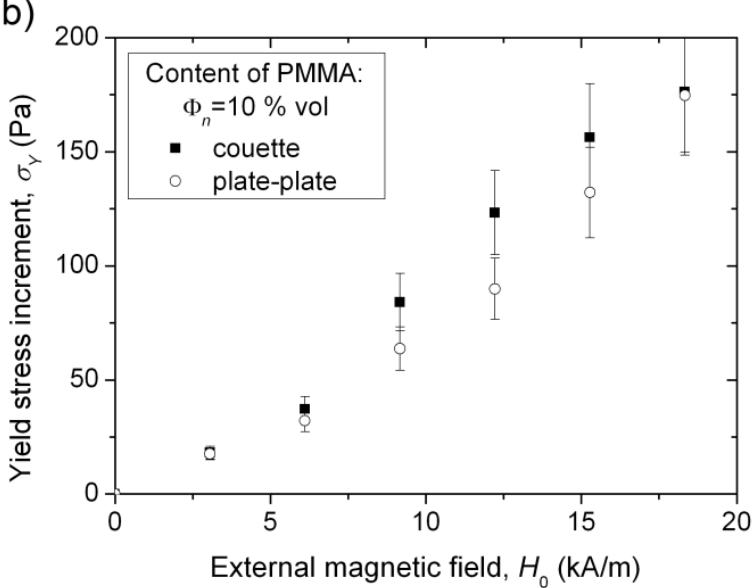

d)

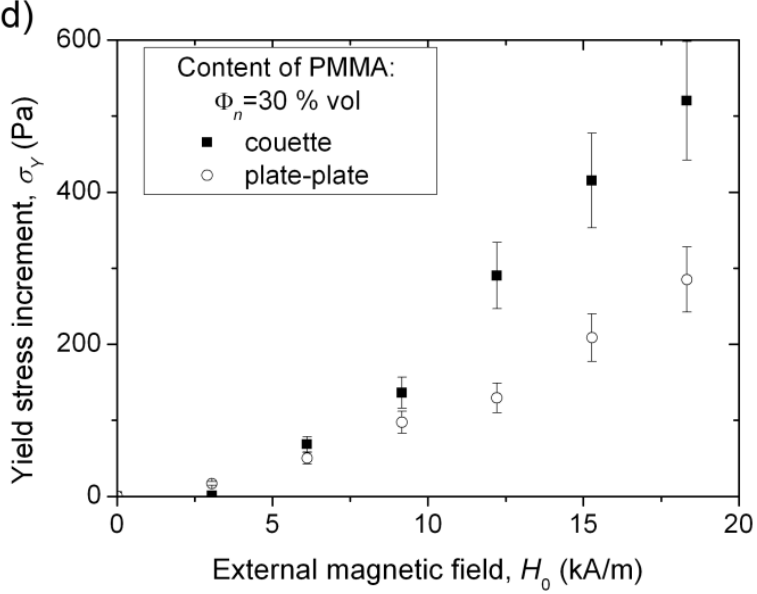

FIG.5. Comparison of the magnetorheological effect observed in plate-plate and cylindrical Couette geometries at the same concentration of iron particles, $\Phi_{m}=0.1$ and four different concentrations of PMMA particles, namely $\Phi_{n}=0$ (a), $\Phi_{n}=0.1$ (b), $\Phi_{n}=0.2$ (c) and $\Phi_{n}=0.3$ (d). The differences between the curves of both geometries increased with $\Phi_{n}$.

\section{THEORY AND DISCUSSION}

\section{A. Qualitative interpretation}

The analysis of the obtained experimental results allows us to formulate the two main questions of the present work, namely: (a) What is the possible mechanism behind the enhancement of the MR effect by the addition of non-magnetic particles to a suspension of magnetic particles? (b) Why is this enhancement appreciably higher when the magnetic field is oriented along the vorticity (cylindrical Couette) rather than along the velocity gradient (plateplate)? In this section, we shall first give a qualitative answer to both questions, followed by the development of a theoretical model to provide a more quantitative interpretation.

Regarding question (a) we must keep in mind that whatever the composition of the suspension-i .e. with or without non-magnetic particles- the dynamic yield stress arises from hydrodynamic dissipation on the field-induced particle aggregates upon shear $[13,15]$. In addition to it, magnetic interactions between aggregates may induce stochastic fluctuations of 
their orientation and result in a supplementary contribution to the yield stress, coming from stochastic interaction torques. These fluctuations may be regarded as an effective rotary diffusion process, which is likely responsible for the unexpectedly high yield stress when the magnetic field is parallel to either the suspension velocity or the vorticity [19, 28].

Our main hypothesis is that under shear flow, the non-magnetic particles may collide with the aggregates of magnetic particles and impart them supplementary fluctuations of their orientations. The importance of such collisions should not be underestimated, taking into account the relatively high concentrations and large size of the PMMA particles used in this work -diameter of the same order of magnitude as the magnetic aggregate thickness as shown in Fig. 1. Clearly, the collision rate, and consequently, the rotary diffusivity of the aggregates, should be a growing function of the volume fraction of non-magnetic particles, $\Phi_{n}$. When $\Phi_{n}$ increases, fluctuations of the aggregate orientation become stronger and two effects take place: (i) The aggregates become more misaligned with the flow, which induces a stronger viscous dissipation (either viscous or hydrodynamic). (ii) The stochastic torque -exerted on the aggregates by multiple collisions with the non-magnetic particles-increases, which results in an increase of the respective stress contribution -the so-called "diffusion stress". Both effects contribute to the increase of the stress level in general, and both of them increase with the volume fraction of PMMA, which would explain the enhancement of the MR effect in bicomponent suspensions.

Concerning question (b), we can also find an answer based on the hypothesis of collisions among the magnetic aggregates and the non-magnetic particles. In cylindrical Couette geometry, the magnetic field tend to orientate the aggregates along the vorticity, which minimizes the viscous dissipation. Consequently, in this particular geometry, depicted in Fig. 6(a), the aggregate orientation distribution and the yield stress are principally governed by the stochastic interactions among the aggregates and the non-magnetic particles, and among the aggregates themselves. On the other hand, in plate-plate geometry, shown in Fig. 6(b), the magnetic torque misaligns the aggregates from the flow direction, which increases the hydrodynamic dissipation and results in a high hydrodynamic stress. In this second case, stochastic interactions are expected to be only a supplementary factor affecting the aggregate orientation and the suspension rheology. These are the reasons for which the effect of collisions with the non-magnetic particles on the orientation distribution and on the suspension yield stress appeared to be more important in cylindrical Couette geometry rather than in plate-plate one. 

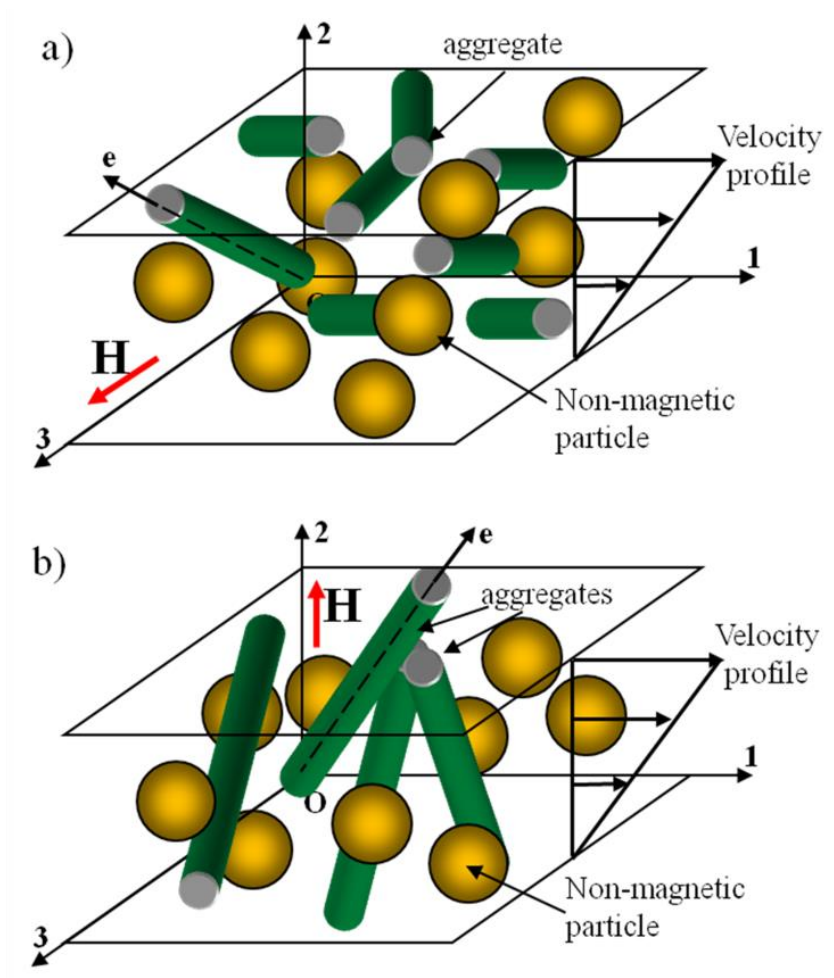

FIG. 6 (color online). Sketch of the problem geometry. The external magnetic field provokes the appearance of aggregates of magnetic particles which are surrounded by a suspension of non-magnetic particles in the liquid carrier. The magnetic field $\mathbf{H}$ is oriented either parallel to the plates along the vorticity - i.e., cylindrical Couette geometry (a) or perpendicularly to the plates -i.e., plate-plate geometry (b).

\section{B. Stochastic interactions and rotational diffusion}

In order to give a quantitative answer to the above stated-questions we shall provide here an expression for the yield stress in bi-component suspensions which takes into account the influence of collisions among the non-magnetic particles and the magnetic aggregates that result in an additional rotary diffusion process. For this purpose we consider a bi-component suspension subjected to a simple shear flow between two infinite plates in the presence of an external magnetic field, $\mathbf{H}_{0}$, oriented either perpendicularly to the plates -configuration similar to the plate-plate geometry- or parallel to the plates along the vorticity -similarly to cylindrical Couette geometry. Both possibilities are shown in the sketches of Figs. 6(a) and 6(b). The relationship between $\mathbf{H}_{0}$, and the internal magnetic field $\mathbf{H}$, is described in the Appendix. The applied magnetic field provokes the appearance of aggregates of magnetic particles, which are surrounded by a suspension of non-magnetic particles in the liquid carrier. The axes of the Cartesian reference frame, "1", "2" and " 3 ", are oriented along the fluid velocity, the velocity gradient and the vorticity respectively. The aggregate orientation is described by a unit vector $\mathbf{e}$, oriented along the aggregate major axis. The orientation distribution is described by secondand fourth-order tensors, $\left\langle e_{i} e_{k}\right\rangle$ and $\left\langle e_{i} e_{k} e_{l} e_{m}\right\rangle$ respectively. These tensors are constructed by the basic projections of the vector $\mathbf{e}$, and are called the statistical moments of the orientation distribution function, or, briefly, statistical moments.

As mentioned above, misalignments of a given aggregate from its equilibrium orientation are induced by magnetic forces exerted by the neighboring aggregates. Since the aggregates are irregularly spaced and polydisperse in size, the forces and torques (interaction 
torques) that they exert on their neighbors vary in a stochastic manner when they displace relative to each other in a shear flow. This mechanism leads to random oscillations of their orientation and can be described as a rotational diffusion process with a diffusion constant, $D_{m}$, defined by a random walk model as follows [1]:

$$
D_{m} \propto\left\langle\omega^{2}\right\rangle \Delta t \propto \frac{\left\langle T_{i n t}^{2}\right\rangle}{f_{r}^{2} \dot{\gamma}}
$$

where $\left\langle\omega^{2}\right\rangle=\left\langle T_{i n t}{ }^{2}\right\rangle / f_{r}{ }^{2}$ is the mean square angular velocity of the aggregates performing stochastic angular jumps of mean duration $\Delta t \propto \dot{\gamma}^{-1} ;\left\langle T_{\text {int }}{ }^{2}\right\rangle$ is the mean square value of the magnetic interaction torque; $f_{r}=8 \pi \eta_{0} L^{3} /(3 \ln \xi)$ is the rotational friction coefficient of an aggregate of length $2 L$ and radius $A ; \eta_{0}$ is the suspending liquid viscosity and $\xi$ is the dimensionless hydrodynamic screening length.

The neighboring aggregates moving around a given aggregate induce some stochastic variation of the magnetic field at the location of a given aggregate because of irregular spacing between their magnetic poles under shear flow. The stochastic field randomly fluctuates during time, such that $\left\langle\mathbf{H}_{s t}\right\rangle=\mathbf{0}$, while its quadratic mean value is supposed to vary as the square of the suspension magnetization: $\left\langle H_{s t}{ }^{2}\right\rangle \square M^{2}$. The fluctuating field induces a stochastic magnetic torque whose mean square value is given by $\left\langle T_{i n t}{ }^{2}\right\rangle=\left\langle\left[\mathbf{m} \times \mathbf{H}_{\mathrm{st}}\right]^{2}\right\rangle \propto\left(\Phi / \Phi_{a}\right) \mu_{0} \chi_{a}{ }^{2} H^{2} V_{a}$. Here the aggregate magnetic moment and the suspension magnetization are estimated as follows: $|\mathbf{m}|=\mu_{0} \chi_{a} H V_{a}$ and $M=\left(\Phi / \Phi_{a}\right) \chi_{a} H$ with $\mu_{0}=4 \pi \cdot 10^{-7} \mathrm{H} / \mathrm{m}$ being the magnetic permeability of vacuum; $\chi_{a}$ is the aggregate magnetic susceptibility, $V_{a}=2 \pi A^{2} L$ is the aggregate volume, $\Phi$ and $\Phi_{a}$ are the volume fraction of particles in the suspension and the internal volume fraction of aggregates, supposed to be equal to $\pi / 6$ for a simple cubic structure; the ratio $\left(\Phi / \Phi_{a}\right)$ stands for the concentration of aggregates in the suspension. Performing the necessary substitutions we arrive to the following expression for the rotary diffusivity:

$$
D_{m}=\alpha_{1}\left(\frac{\Phi \mu_{0} \chi_{a}{ }^{2} H^{2}}{\Phi_{a} \eta_{0} \beta}\right)^{2} \frac{1}{\dot{\gamma}}
$$

where $\beta=4 r_{e}^{2} /(3 \ln \xi)$ is the form-factor describing the hydrodynamic resistance of the aggregates and coming from the slender body theory [29]; $r_{e}=L / A$ is the aggregate aspect ratio. Because we are able to estimate only the order of magnitude of the stochastic field and of the interaction torque, we need to introduce a dimensionless correction factor $\alpha_{1}$ into Eq. (2) which describes the intensity of the stochastic magnetic interactions between aggregates and which will be taken as an adjustable parameter.

Note that the aggregates can be destroyed by tensile hydrodynamic forces once they are misaligned from the flow or the vorticity direction. More specifically, the aggregate size, and consequently its form-factor $\beta$, is defined by a compromise between the destructive hydrodynamic and magnetic cohesive forces [30,31]. By applying the force balance (whose 
general expression is given in [19]) to both studied configurations (Figs. 6a and 6b), we arrive to the following expression for the form factor:

$$
\beta \equiv \frac{4 r_{e}^{2}}{3 \ln \xi}=\frac{2 \Phi_{a} f_{m}}{\eta_{0} \dot{\gamma} \psi}
$$

where $\psi$ is a numerical factor depending on the orientation state of the suspension and equal to $\left(1-\left\langle e_{3}^{2}\right\rangle\right) /\left(2\left\langle e_{3}^{2}\right\rangle\right)$ for the field parallel to the vorticity (cylindrical Couette geometry, Fig. 6a) and $\left\langle e_{1} e_{2}\right\rangle /\left\langle e_{2}^{2}\right\rangle$ for the field parallel to the velocity gradient (plate-plate geometry, Fig. 6b); $f_{m}$ is the magnetic force between neighboring particles constituting the aggregates, per unit crosssectional area of the particle. The magnetic force $f_{m}$ and the aggregate magnetic susceptibility $\chi_{a}$, intervening into Eqs. (2) and (3) are functions of the magnetic field, their field dependences being given in the Appendix.

The above considered random fluctuations of the aggregate orientation are attributed to long-range magnetic interactions between aggregates. As already stated, such a field-induced diffusion may be substantially enhanced by the collisions of the magnetic aggregates with the non-magnetic particles. The diffusivity of this second collision-induced diffusion mechanism, $D_{c}$, is supposed to be linear with both the concentration of non-magnetic particles, $\Phi_{n}$, and with the collision rate, and thus, with the shear rate $\dot{\gamma}$. Both diffusion mechanisms are supposed to be additive, so that the effective diffusion constant would be the sum of two respective diffusivities:

$$
D_{r}=D_{m}+D_{c}=\alpha_{1}\left(\psi \frac{\Phi_{m} \mu_{0} \chi_{a}^{2} H^{2}}{2 \Phi_{a}^{2} f_{m}}\right)^{2} \dot{\gamma}+\alpha_{2} \Phi_{n} \dot{\gamma}
$$

The first term of Eq. (1) is obtained by replacing the form-factor $\beta$ in Eq. (2) by the expression (3). As in the case of magnetically induced diffusion, we are unable to provide an exact relationship for the collision-induced diffusivity $D_{c}$. This quantity is therefore defined up to a dimensionless phenomenological constant $\alpha_{2}$, which describes the intensity of collisioninduced angular fluctuations and depends on microscopic details of the process discarded in the present model. This constant is taken as the second adjustable parameter of the model.

\section{Orientation distribution}

The orientation state of the aggregates is described by the equation of evolution of the second statistical moments, $\left\langle e_{i} e_{k}\right\rangle$, which is conventionally derived by multiplying the FokkerPlanck equation for the orientation distribution function by $e_{i} e_{k}$, and averaging over all possible orientations. In the case of long aggregates possessing an induced magnetic moment, this equation reads $[19,28,32]$ : 


$$
\begin{aligned}
& \frac{d\left\langle e_{i} e_{k}\right\rangle}{d t}=\left[\omega_{i l}\left\langle e_{l} e_{k}\right\rangle-\left\langle e_{i} e_{l}\right\rangle \omega_{l k}\right]+\left[\gamma_{i l}\left\langle e_{l} e_{k}\right\rangle+\left\langle e_{i} e_{l}\right\rangle \gamma_{l k}\right]-2\left\langle e_{i} e_{k} e_{l} e_{m}\right\rangle \gamma_{l m} \\
& +\frac{\mu_{0} H^{2}}{\eta_{0} \beta} \frac{\chi_{a}^{2}\left(1-\Phi / \Phi_{a}\right)}{2+\chi_{a}\left(1-\Phi / \Phi_{a}\right)}\left[h_{i} h_{l}\left\langle e_{l} e_{k}\right\rangle+h_{k} h_{l}\left\langle e_{l} e_{i}\right\rangle-2\left\langle e_{i} e_{k} e_{l} e_{m}\right\rangle h_{l} h_{m}\right]+2 D_{r}\left[\delta_{i k}-3\left\langle e_{i} e_{k}\right\rangle\right]
\end{aligned}
$$

where $t$ is the time; $\gamma_{i k}=(1 / 2) \cdot\left(\partial v_{i} / \partial x_{k}+\partial v_{k} / \partial x_{i}\right)$ and $\omega_{i k}=(1 / 2) \cdot\left(\partial v_{i} / \partial x_{k}-\partial v_{k} / \partial x_{i}\right)$ are the rate-of-strain and vorticity tensors, respectively; $h_{i}$ is the $i$-th component of the unit vector $\mathbf{h}$ oriented along the internal magnetic field $\mathbf{H} ; \delta_{i k}$ is the Kronecker delta. In our case, we have only two non-zero components of the rate-of-strain and vorticity tensors, $\gamma_{12}=\gamma_{21}=\omega_{12}=-\omega_{21}=\dot{\gamma} / 2$ and the one non-zero component of the field unit vector: either $h_{3}=1$ for the field oriented along the vorticity (Fig. 6(a)) or $h_{2}=1$ for the field oriented along the velocity gradient (Fig. 6(b)).

The last reduces to the following system of algebraic equations for the steady state upon application of the quadratic closure approximation [33], $\left\langle e_{i} e_{k} e_{l} e_{m}\right\rangle \equiv\left\langle e_{i} e_{k}\right\rangle\left\langle e_{l} e_{m}\right\rangle$, and using expressions (3) and (4) for the form-factor $\beta$ and the rotary diffusivity respectively:

$$
\left\{\begin{array}{l}
\left\langle e_{1} e_{2}\right\rangle-\left\langle e_{1}^{2}\right\rangle\left\langle e_{1} e_{2}\right\rangle-C_{2} \psi\left\langle e_{1}^{2}\right\rangle \psi_{11}+\left(C_{1} \psi^{2}+\alpha_{2} \Phi_{n}\right)\left(1-3\left\langle e_{1}^{2}\right\rangle\right)=0 \\
-\left\langle e_{2}^{2}\right\rangle\left\langle e_{1} e_{2}\right\rangle-C_{2} \psi\left\langle e_{2}^{2}\right\rangle \psi_{22}+\left(C_{1} \psi^{2}+\alpha_{2} \Phi_{n}\right)\left(1-3\left\langle e_{2}^{2}\right\rangle\right)=0 \\
-\left\langle e_{3}^{2}\right\rangle\left\langle e_{1} e_{2}\right\rangle-C_{2} \psi\left\langle e_{3}^{2}\right\rangle \psi_{33}+\left(C_{1} \psi^{2}+\alpha_{2} \Phi_{n}\right)\left(1-3\left\langle e_{3}^{2}\right\rangle\right)=0 \\
\left\langle e_{2}^{2}\right\rangle-2\left\langle e_{1} e_{2}\right\rangle^{2}-C_{2} \psi\left\langle e_{1} e_{2}\right\rangle \psi_{12}-6\left(C_{1} \psi^{2}+\alpha_{2} \Phi_{n}\right)\left\langle e_{1} e_{2}\right\rangle=0
\end{array}\right.
$$

where

$$
C_{1}=\alpha_{1}\left[\Phi_{m} \mu_{0} \chi_{a}^{2} H^{2} /\left(2 \Phi_{a}^{2} f_{m}\right)\right]^{2}
$$

and $C_{2}=\mu_{0} \chi_{a}{ }^{2}\left(1-\Phi_{m} / \Phi_{a}\right) H^{2} /\left[2 \Phi_{a} f_{m}\left(2+\chi_{a}\left(1-\Phi_{m} / \Phi_{a}\right)\right)\right]$ are dimensionless factors; the coefficients $\psi_{i k}$ are functions of the second statistical moments and are equal to $\left[\psi_{11}, \psi_{22}, \psi_{33}, \psi_{12}\right]=\left[\left\langle e_{2}^{2}\right\rangle,\left\langle e_{2}^{2}\right\rangle-1,\left\langle e_{2}^{2}\right\rangle, 2\left\langle e_{2}^{2}\right\rangle-1\right]$ for the field oriented along the velocity gradient (Fig.6(b)) and $\left[\psi_{11}, \psi_{22}, \psi_{33}, \psi_{12}\right]=\left[\left\langle e_{3}^{2}\right\rangle,\left\langle e_{3}^{2}\right\rangle,\left\langle e_{3}^{2}\right\rangle-1,2\left\langle e_{3}^{2}\right\rangle\right]$ for the field oriented along the vorticity (Fig. 6(a)).

It is important to remark that, due to the fact that the rotary diffusivity $D_{r}$ is linear in the shear rate (Eq. (4)) and the form-factor $\beta$ is inversely proportional to the shear rate (Eq. (3)), the shear rate vanishes from equations (6) describing the statistical moments at the steady-state condition; the orientation distribution is therefore independent of shear rate, at least in the limit of long aggregates, $r_{e} \square 1$ considered here. This result agrees with the classical models of magnetorheology, discarding any dispersion in aggregate orientation and predicting an angle between the aggregates and the flow independent of shear rate [15, 31]. The system of Eq. (6) is solved numerically with respect to the four unknown second statistical moments, $\left\langle e_{1}^{2}\right\rangle,\left\langle e_{2}^{2}\right\rangle$ ,$\left\langle e_{3}^{2}\right\rangle$ and $\left\langle e_{1} e_{2}\right\rangle$. 
In Fig. 7, we inspect the behavior of the second statistical moments as a function of the volume fraction $\Phi_{n}$ of non-magnetic particles at a fixed content of magnetic ones, $\Phi_{m}=0.1$ and for an intensity of the applied external magnetic field, $H_{0}=18.3 \mathrm{kA} / \mathrm{m}$. At the considered set of free parameters (chosen to provide the best fit to the experimental yield stress, cf. Figs. 4 and 8 ), the orientation state seems to be moderately influenced by collisions with non-magnetic particles but slowly evolves to an isotropic state with an increase of the concentration $\Phi_{n}$. All the statistical moments, exhibit a somewhat stronger variation for the magnetic field oriented along the vorticity (in cylindrical Couette geometry) (Fig. 7(a)), as compared to the case of the field oriented along the velocity gradient (plate-plate geometry) (Fig. 7(b)). This should contribute to a more pronounced effect of the non-magnetic particle content, $\Phi_{n}$, on the stress level in Couette geometry.
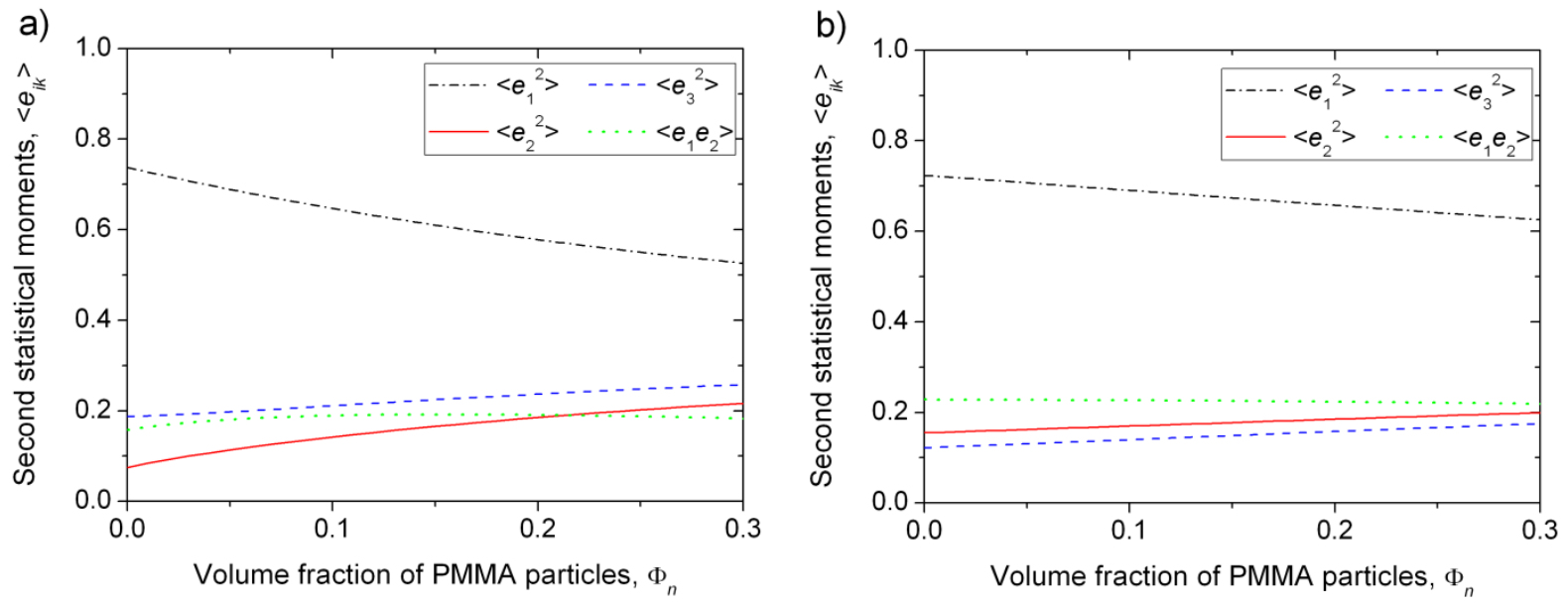

FIG. 7. (Color online) Theoretical dependencies of the second statistical moments on the volume fraction of nonmagnetic particles for the cylindrical Couette (a) and the plate-plate (b) geometries. For both geometries, the intensity of the external magnetic field is $H_{0}=18.3 \mathrm{kA} / \mathrm{m}$ and the concentration of the magnetic particles is $\Phi_{m}=0.1$. The free parameters are chosen to provide the best fit with experimental data on the suspension yield stress (see Figs. 4 and 8) and are equal to $\alpha_{1}=1.5 ; \alpha_{2}=0.2$ for the plate-plate geometry and $\alpha_{1}=0.2 ; \alpha_{2}=0.4$ for the cylindrical Couette geometry.

\section{Suspension stress}

The stress tensor developed in the non-Brownian bi-component magnetic suspension can be estimated assuming that the long aggregates of magnetic particles are immersed in an effective medium composed of a homogeneous suspension of non-magnetic (PMMA) particles dispersed in a suspending liquid of viscosity $\eta_{0}$. The effective viscosity of such a medium can be estimated using the Krieger-Dougherty equation for concentrated hard sphere suspensions [27]: $\eta_{e}=\eta_{0}\left(1-\Phi_{n} / \Phi_{\max }\right)^{-2.5 \Phi_{\max }}$, with $\Phi_{\max } \approx 0.64$ being the random close packing fraction of the hard spheres. Under such condition, we may use the well-known expression for the stress tensor in the semi-dilute suspensions of axisymmetric particles [34], which, being applied to the case of long aggregates with induced magnetic moments, reads [20, 29]: 


$$
\begin{aligned}
\sigma_{i k} & =-p \delta_{i k}+2 \eta_{e} \gamma_{i k}+\frac{\Phi_{m}}{\Phi_{a}} \eta_{e}\left\{4 \gamma_{i k}+\frac{\beta}{2}\left[\left\langle e_{i} e_{k} e_{l} e_{m}\right\rangle-\frac{1}{3} \delta_{i k}\left\langle e_{l} e_{m}\right\rangle\right] \gamma_{l m}\right\}+ \\
& +\frac{\Phi_{m}}{\Phi_{a}} \mu_{0} H^{2} \frac{\chi_{a}{ }^{2}\left(1-\Phi_{m} / \Phi_{a}\right)}{2+\chi_{a}\left(1-\Phi_{m} / \Phi_{a}\right)}\left[\left\langle e_{i} e_{k} e_{l} e_{m}\right\rangle h_{l} h_{m}-h_{i} h_{l}\left\langle e_{l} e_{k}\right\rangle\right]+\frac{\Phi_{m}}{\Phi_{a}} \beta \eta_{e} D_{r}\left[3\left\langle e_{i} e_{k}\right\rangle-\delta_{i k}\right]
\end{aligned}
$$

where $p$ is the pressure in the suspension and the solvent viscosity $\eta_{0}$ appearing in the original expression for the stress tensor has been replaced by the effective medium viscosity $\eta_{e}$. Replacing the diffusion constant and the form-factor $\beta$ by appropriate expressions (Eqs. (3) and (4)), the shear rate vanishes from the last three terms of Eq. (7) and we recover the Bingham rheological law for the shear stress ( $\sigma_{12}$ component of the stress tensor): $\sigma_{12}=\sigma_{Y}+\eta \dot{\gamma}$ with the plastic viscosity $\eta=\eta_{e}\left(1+2 \Phi_{m} / \Phi_{a}\right)$. The dynamic yield stress is thus given by the following expression, valid for both considered geometries:

$$
\begin{aligned}
\sigma_{Y}= & \Phi_{m} f_{m} \frac{\left\langle e_{1} e_{2}\right\rangle^{2}}{\psi}+\frac{\Phi_{m}}{\Phi_{a}} \cdot \frac{\chi_{a}{ }^{2}\left(1-\Phi_{m} / \Phi_{a}\right)}{2+\chi_{a}\left(1-\Phi_{m} / \Phi_{a}\right)} \mu_{0} H^{2}\left\langle e_{1} e_{2}\right\rangle \psi_{11} \\
& +\frac{3}{2} \alpha_{1} \frac{\Phi_{m}{ }^{3}}{\Phi_{a}{ }^{4}} \frac{\left(\mu_{0} \chi_{a}{ }^{2} H^{2}\right)^{2}}{f_{m}} \psi\left\langle e_{1} e_{2}\right\rangle+6 \alpha_{2} \Phi_{m} \Phi_{n} f_{m} \frac{\left\langle e_{1} e_{2}\right\rangle}{\psi}
\end{aligned}
$$

where the quantities $\left\langle e_{1} e_{2}\right\rangle, \psi$ and $\psi_{11}$, characterizing the aggregate orientation state are found from solution of Eqs. (6). The first term on the right-hand side of Eq. (8) is the hydrodynamic part of the aggregate stress. The second term corresponds to the external torque exerted on the aggregates by the magnetic field $H$. The third term is the diffusion stress coming from the stochastic magnetic torques exerted on the aggregates by neighboring aggregates. Finally the fourth term stands for the diffusion stress coming from the collisions between aggregates and non-magnetic particles. This last component marks the contribution of the non-magnetic particles to the suspension yield stress and depends on the applied magnetic field, being proportional to the magnetic force between particles $f_{m}$. This is not surprising because the aggregate aspect ratio is an increasing function of the applied field, $r_{e}^{2} \propto f_{m} / \dot{\gamma}$, and longer aggregates induce a stronger viscous dissipation when subjected to random collisions. As a result, the collision-induced stress scales as $\sigma_{c} \propto r_{e}^{2} D_{c} / \dot{\gamma} \propto f_{m}$ with the diffusivity $D_{c} \propto \dot{\gamma}$ (cf. Eq. (4)). Note that deriving Eq. (8), we did not take into account eventual colloidal interactions in the suspension, which result in an off-state yield stress of the real suspension. Accordingly, the yield stress presented in Eq. (8) should be considered as the increment of the yield stress. Finally, to explain the appearance of the yield stress in both considered geometries, we must recall that the aggregate aspect ratio is a decreasing function of the shear rate, $r_{e} \propto \dot{\gamma}^{-1 / 2}$ (Eq. (3)) and the rotary diffusivity is proportional to the shear rate, $D_{r} \propto \dot{\gamma}$ (Eq. (4)). Therefore both the hydrodynamic stress, $\sigma_{H} \propto r_{e}^{2} \dot{\gamma}$, and the diffusion stress, $\sigma_{D} \propto r_{e}^{2} D_{r}$, appear to be independent of the shear rate, at least in the limit of long aggregates, $r_{e} \square 1$, not spanning the rheometer gap [15-31]. Because of such independence, the aggregate stress is considered to be the dynamic yield stress (Eq. (8)) of the suspension.

\section{E. Comparison with experiments}


Both the theoretical and experimental increments of the yield stress show a monotonic growth with the field. Such effect can be attributed to increasing magnetic interactions between the magnetic particles inside the aggregates -Fig. 4. Curiously, the field dependency becomes sub-linear at magnetic fields $H_{0}>20 \mathrm{kA} / \mathrm{m}$ in the case of the cylindrical Couette geometry (Fig. 4(a)). Such a behavior can be explained by a similar sub-linear field dependency of the magnetic force $f_{m}$, as inferred from finite element method simulations. This effect is not observed in the plate-plate geometry at the same range of magnetic fields (Fig. 4(b)). This is likely because the magnetic component of the yield stress (second term in Eq. (8), proportional to $H^{2}$ ) is more important in this particular geometry than in cylindrical Couette geometry, and masks the sublinear trend of $f_{m}$.

As expected, the theory predicts an increase of the yield stress increment with the content of non-magnetic particles for both geometries. The two free parameters $\alpha_{1}$ and $\alpha_{2}$, characterizing the intensity of the field-induced and collision-induced angular fluctuations of the aggregate orientation, are used to fit the theory to the experimental curves. The first parameter, $\alpha_{1}$, is fitted to the experimental curve corresponding to $\Phi_{n}=0$. The second parameter, $\alpha_{2}$, is fitted to the experimental curves at $\Phi_{n}>0$ keeping the parameter $\alpha_{1}$ fixed. The best fit corresponds to the following values of the free parameters: $\alpha_{1}=1.5 ; \alpha_{2}=0.2$ for the plate-plate geometry and $\alpha_{1}=0.2 ; \alpha_{2}=0.4$ for the cylindrical Couette geometry. Therefore we can see that the collision contribution to the rotary diffusivity, $\alpha_{2}$, is more important in Couette geometry, which explains the stronger effect of the PMMA addition in this case.

The effect of adding non-magnetic particles on the suspension rheology can be better analyzed in Fig. 8 where the dependencies of the yield stress increment on the concentration of non-magnetic particles are plotted. As already noticed, the yield stress increment exhibits a stronger increase with the concentration of PMMA in the cylindrical Couette geometry. An alternative measure of the effect of the non-magnetic particles on the yield stress is the magnitude, $\left[\sigma_{Y}\left(\Phi_{n}\right)-\sigma_{Y}(0)\right] / \sigma_{Y}(0)$, which describes the gain of the MR effect due to the nonmagnetic particles, where $\sigma_{Y}(0)$ is the yield stress increment of the suspension without PMMA. This magnitude is plotted in the insets of Fig. 8 as a function of the content of non-magnetic particles, $\Phi_{n}$. Both experiments and theory show a monotonic increase of the MR effect with $\Phi_{n}$ in both geometries. It is worth now to recall that, according to our theory, the non-magnetic particles influence the yield stress by the two following mechanisms: (a) They modify the orientation distribution of the aggregates due to collisions with them (see Fig. 8). (b) They cause a supplementary viscous dissipation by enhancing random fluctuations of aggregate orientation -i.e., last term of Eq. (8). Analyses show that the second mechanism appears to be dominant in the yield stress enhancement at the considered experimental conditions. 

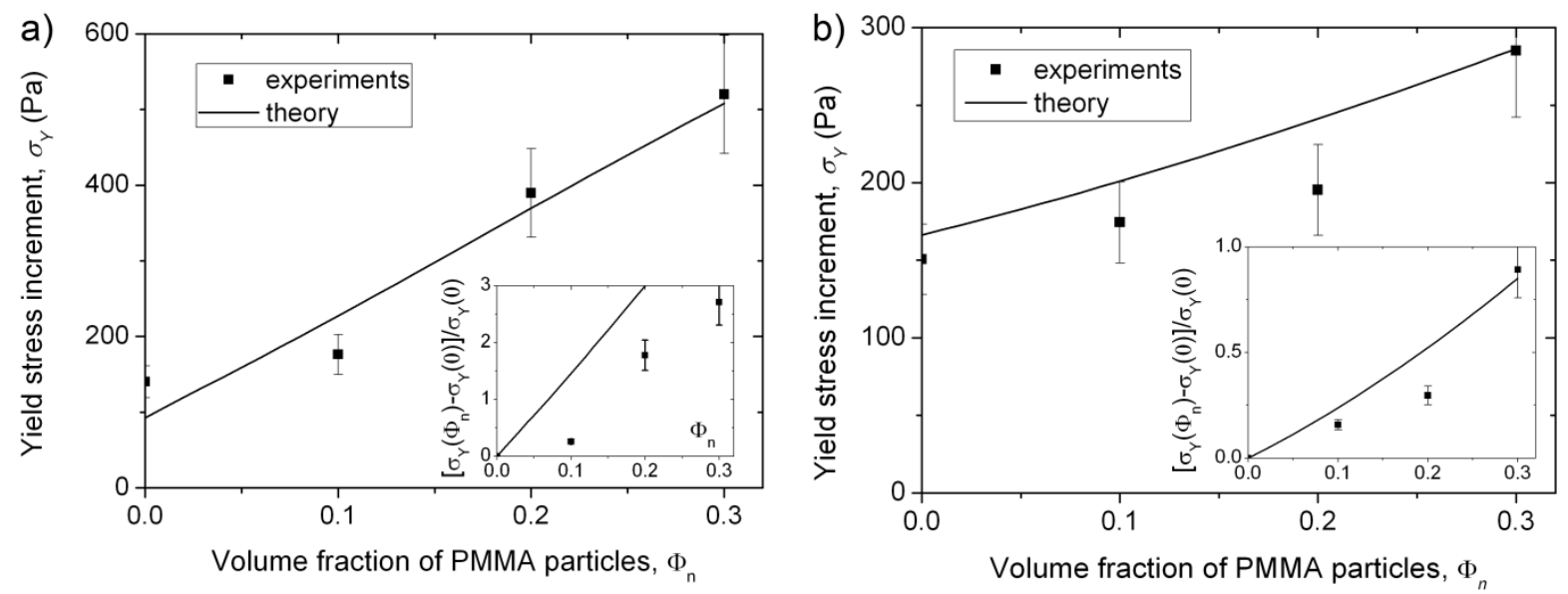

FIG. 8. Theoretical and experimental dependencies of the yield stress increment on the concentration of the nonmagnetic particles, $\Phi_{n}$, at a fixed concentration of the magnetic ones, $\Phi_{m}=0.1$, for cylindrical Couette (a) and plateplate (b) geometries. The external magnetic field is $H_{0}=18.3 \mathrm{kA} / \mathrm{m}$ in both cases. The free parameters are $\alpha_{1}=1.5$; $\alpha_{2}=0.2$ for the plate-plate geometry and $\alpha_{1}=0.2 ; \alpha_{2}=0.4$ for the cylindrical Couette geometry. Insets of both figures show the gain of the MR effect as function of the concentration of non-magnetic particles. Similar results were obtained for other fields, not shown here for simplicity.

Finally note that, despite its simplicity, the present model captures the enhancement of the MR effect in bi-component suspensions without specific interactions between both species. Because of the opacity of the suspensions, it seems to be quite difficult to verify the hypothesis of the collision-induced fluctuations using classical optical microscopy. Direct numerical simulations might also elucidate the role of the non-magnetic particles on the structure of the flowing suspension and give more precise expressions for the rotary diffusivities as a function of the concentration and the size ratio of both species of particles. Nevertheless, and in contrast to our experiments, the existing numerical results of refs. [22,23] have revealed only a moderate (a few dozens of percents) enhancement of the MR effect by the addition of non-magnetic particles. This is probably because a low-shear regime was considered in simulations. In this regime, the moving aggregates span the channel width, which likely hinders their orientation fluctuations. In our study, we have dealt with higher shear rates, where the aggregates are notgap spanning and have more freedom for interactions with their neighbors as well as with nonmagnetic particles.

\section{CONCLUSIONS}

In this work we have shown that bi-component suspensions consisting of magnetic iron- and non-magnetic -poly(methylmethacrylate), PMMA- particles displayed an enhancement of the magnetorheological (MR) effect with respect to a suspension of just iron particles with the same volume fraction of magnetic material, both in cylindrical Couette and plate-plate geometries. Such an enhancement took place even when presumably there was no adhesion of iron particles onto PMMA ones, because the former particles were covered with a surfactant layer. Magnetization measurements showed that the magnetic permeability was not affected by the addition of PMMA which is also attributable to the absence of adsorption between both types of particles. We conclude therefore that the MR effect enhancement does 
not come from an increase of the magnetic interactions, something which would take place if the iron particles formed a shell structure around the non-magnetic PMMA particles [25].

We have explained the observed phenomenon under the hypothesis of collisions among the non-magnetic particles and the field-induced aggregates of magnetic particles. Such collisions are supposed to give rise to an enhancement of the fluctuations of the aggregate orientation that increases with the volume fraction of non-magnetic particles. This process contributes to augment the total level of stress in the suspension and therefore, the MR effect. We have shown that in cylindrical Couette geometry, this mechanism is predominant in comparison to the case of plate-plate geometry, in which it plays a minor role. As a result, the improvement of the MR effect in bi-component suspensions is more noticeable for cylindrical Couette geometry, in agreement with experiments.

\section{ACKNOWLEDGEMENTS}

We would like to acknowledge Dr. G. Bossis for helpful discussions. This work has been supported by Project "Factories of the Future" (Grant No. 260073, DynExpert FP7). In addition, L. Rodríguez-Arco acknowledges financial support by Secretaría de Estado de Educación, Formación Profesional y Universidades (MECD, Spain) through its FPU and Estancias Breves programs.

\section{APPENDIX. RELATIONSHIP BETWEEN THE INTERNAL, H, AND THE EXTERNAL, Ho, MAGNETIC FIELDS}

In the case of the magnetic field oriented along the vorticity (cylindrical Couette geometry, Fig. 6(a)), the internal field is equal to the external applied magnetic field $H_{0}$. In the case of the magnetic field perpendicular to the walls (plate-plate geometry, Fig. 6(b)), the internal field is related to the external field $H_{0}$ through the following expression:

$$
H=\frac{H_{0}}{\mu_{22}}=\frac{H_{0}}{\mu_{\square}\left\langle e_{2}^{2}\right\rangle+\mu_{\perp}\left(1-\left\langle e_{2}^{2}\right\rangle\right)}
$$

where $\mu_{22}$ is the diagonal component (along the field axis " 2 ") of the magnetic permeability tensor of the suspension; $\mu_{\square}=1+\chi_{a} \Phi_{m} / \Phi_{a} \quad$ and $\mu_{\perp}=\left(2+\chi_{a}\left(1+\Phi_{m} / \Phi_{a}\right)\right) /\left(2+\chi_{a}\left(1-\Phi_{m} / \Phi_{a}\right)\right)$ are the components of the magnetic permeability of the suspension whose aggregates are, respectively, aligned or perpendicular to the applied field.

The aggregate magnetic susceptibility $\chi_{a}$ and the magnetic force $f_{m}$ (intervening into Eqs. (3), (4) and (8)) are calculated as a function of the magnetic field $H$ using finite element simulations $[18,28]$ The simulation results for $f_{m}$ and $\chi_{a}$, have been fitted by the following expressions, valid in the range of the magnetic field intensities, $0 \leq H \leq 30 \mathrm{kA} / \mathrm{m}$ : $f_{m}(H)=\left(6.66 \cdot 10^{4}\left(H / M_{S}\right)^{2}-6.32 \cdot 10^{3}\left(H / M_{S}\right)+168\right) \mu_{0} H^{2} \quad$ and $\chi_{a}(H)=-7.67 \cdot 10^{2}\left(H / M_{S}\right)^{2}-50.9\left(H / M_{S}\right)+9.29$, with $M_{S}=1.36 \cdot 10^{6} \mathrm{~A} / \mathrm{m}$ being the saturation magnetization of the carbonyl iron particles. 


\section{References}

[1] G.M. Van de Ven, Colloidal hydrodynamics (Academic Press Limited, London, 1989).

[2] P. Heitjans and J. Kärger, Diffusion in Condensed Matter. Methods, Materials, Models (Springer, Berlin, 2005).

[3] Z. Cheng and T.G. Mason, Phys. Rev. Lett. 90, 018304 (2003).

[4] V. Degiorgio and R. Piazza, Phys. Rev. E 52, 2707 (1995).

[5] A. Elafif, M. Grmela, and G. Lebon, J. Non-Newton. Fluid 86, 253 (1999).

[6] S. Jabbari-Farouji, G. H. Wegdam, and Daniel Bonn, Phys. Rev. E 86, 041401 (2012).

[7] B.H. Erné, K. Butter, B. W. M. Kuipers, and G. J. Vroege, Langmuir 19, 8218 (2003).

[8] J. M. Ginder, Phys. Rev. E 47, 5 (1993).

[9] A. Zubarev, Colloid J. 75, 59 (2013).

[10] J.C. Bacri, A. Cebers, A. Bourdon, G. Demouchy, B.M. Heegaard, B.Kashevsky, and R. Perzynski, Phys. Rev. E 52, 4 (1995).

[11] J.E. Martin, J. Odinek, T. C. Halsey, and R. Kamien, Phys. Rev. E 57, 1 (1998).

[12] J.P. Segovia-Gutiérrez, J. de Vicente, R. Hidalgo-Álvarez, and A. Puertas, Soft Matter 9, 6970 (2013).

[13] G. Bossis, O. Volkova, S. Lacis, and A. Meunier, in Magnetorheology: Fluids, Structures and Rheology, edited by S. Odenbach (Springer-Verlag, Berlin, 2002), p. 202.

[14] B.J. Park, F.F. Fang, and H.J. Choi, Soft Matter 6, 5246 (2010).

[15] Z.P. Shulman and W. I. Kordonsky, Magnetorheological effect (Nauka i Tehnika, Minsk, 1982) (in Russian).

[16] J. Takimoto, H. Takeda, Y. Masubuchi, and K. Koyama, Int. J. Mod. Phys. B 13, 2028 (1999).

[17] G. Bossis, S. Lacis, A. Meunier, and O. Volkova, J. Magn. Magn. Mater. 252, 224 (2002).

[18] P. Kuzhir, G. Bossis, V. Bashtovoi, and O. Volkova, J. Rheol. 47, 1385 (2003).

[19] P. Kuzhir, C. Magnet, G. Bossis, A. Meunier, and V. Bashtovoi, J. Rheol. 55, 1297 (2011).

[20] M.T. López-López, J. de Vicente, F. González-Caballero, and J.D.G. Durán Colloid. Surface A 264, 75 (2005).

[21] M.T. López-López, A. Gómez-Ramírez, J.D.G. Durán, and F. González-Caballero, Langmuir 24, 7076 (2008).

[22] J. C. Ulicny, K. S. Snavely, M. A. Golden, and D. J. Klingenberg, Appl. Phys. Lett. 96, 231903 (2010).

[23] D.J. Klingenberg and J.C. Ulicny, Int. J. Mod. Phys. B 25, 911 (2011).

[24] M.L. Levin, D.E. Polesskii, and I.V. Prokhorov, J. Eng. Phys. Thermophys. 70, 769 (1997).

[25] L. Rodríguez-Arco, M.T. López-López, P. Kuzhir, and J.D.G. Durán, Soft Matter 9, 5726 (2013). 
[26] L. Rodríguez-Arco, M.T. López-López, P. Kuzhir, G. Bossis, and J.D.G. Durán, ACS Appl. Mater. Inter. 5, 12143 (2013).

[27] R. G. Larson, The Structure and Rheology of Complex Fluids (Oxford University Press, New York, 1999), p. 353.

[28] P. Kuzhir, C. Magnet, L. Rodríguez-Arco, M.T. López-López, A. Meunier, A. Zubarev, and G. Bossis, J. Rheol. (submitted).

[29] G.K. Batchelor, J. Fluid. Mech. 46, 813 (1971).

[30] Z.P. Shulman, V.I. Kordonsky, E.A. Zaltsgendler, I.V. Prokhorov, B.M. Khusid, and S.A. Demchuk, Int. J. Multiphase Flow 12, 935 (1986).

[31] J.E. Martin and R.A. Anderson, J. Chem. Phys. 104, 4814 (1996).

[32] V.N. Pokrovskiy, Statistical mechanics of diluted suspensions (Nauka, Moscow, 1978).

[33] M. Doi and S.F. Edwards, The Theory of Polymer Dynamics (Oxford University Press, New York, 1986).

[34] H. Brenner, Int. J. Multiphase Flow 1, 195 (1974). 